\title{
To Evaluate The Efficacy Of Crusade Execution In Palisade - Disquieting Arthritic Talus With And Without Alkane Swell Bath
}

\author{
Dr Jopex Lange , Dr Nikolaes Heinsius, Dr Rein Strikwerda \\ Groningenty University Of Medical Sciences Netherlands
}

\begin{abstract}
Crusade systems are much of the time utilized by physiotherapists to lessen torment, improve joint development and encourage come back to exercises after damage. The goal of this examination was to investigate contrasts in the viability of crusade Execution in Post-Traumatic firm lower leg with and without Paraffin Wax Bath. Execution : Thirty seven patients of Post Traumatic firm lower leg were enlisted for the examination at Sajid Physiotherapy and Rehabilitation focus, Multan from March 2011 to February 2013. It was a randomized controlled preliminary and the patients with equivalent evaluations of seriousness were put in charge and study gatherings. Gathering A had nineteen patients and Group B had 18 patients. The consideration criteria were age extend from 20-60 years, torment, loss of ROM, with history of injury and break of lower leg. The patients with comparable protests yet with careful treatment were prohibited. Gathering A was given preparation methods with paraffin wax shower while bunch B was treated without paraffin wax shower. Improvement was seen by EscolaPaulista de Medicinal Range of Motion (EPM-ROM) scale and visual simple scale (VAS). Following ten weeks of treatment, the patients were reconsidered by an orthopedic specialist and a Physiotherapist for their side effects and ROM. t-test was connected to look at result between two gatherings and $p<0.05$ was viewed as factually critical.
\end{abstract}

Keywords: crusade procedures, Paraffin wax shower, Stiff lower leg. 


\section{Introduction}

The lower leg is especially helpless against injury. The bones of the lower leg are subcutaneous. The delicate tissue envelope comprises of just skin, ligament, and neurovascular structures foremost, parallel, and average to the joint. Just in the back quadrant is there an unobtrusive solid envelope. What's more, the lower leg joint does not endure disfigurement or articular confusion after injury. Indications of a break incorporate quick and extreme agony, swelling, wounding, delicacy to the touch, failure to put weight on the foot and visual deformation. The post-horrible hardened lower leg by and large prompts neglect of foot work, because of confined scope of movement and loss of muscle quality. The physical advisors restore the patients with post-awful firm lower leg by joint assembly systems, extending and reinforcing works out. On the off chance that the patients are not restored, they will create contractures and foot goes to valgus disfigurement and will bring about a place of dysfunction. 2 The exercise based recuperation plan of consideration depends on physical assessment, incorporates assessment of ROM, muscle quality, edema, net sensation, bone mending, and grips.

The improvement in joint ROM is the key segment of active recuperation the executives, due to musculotendinous snugness. Joint activation or control includes inactively moving the joints of the human body in a manner that can't be cultivated by dynamic development (developments you perform yourself). It includes little sliding developments of the bone surfaces into the bearing of confinement. Interpretations, floats notwithstanding moving movements are evaluated and remedied in during a common joint assembly treatment. 4 There are a few distinct evaluations of joint activation going from evaluation one to five. Evaluations one, two and three are delicate developments intended to anticipate grip and lessen torment. Evaluation four activations, the most generally performed assembly is an extending movement performed at the joint's end run. Evaluation five preparations is intended to reestablish portability to the joint, additionally have the additional advantage of agony decrease because of a rebuilding of typical joint arthrokinematics.

diversion between the articular surfaces of hand joints to oversee torment, break attachments, and improve joint ROM.5 There are numerous clinical reports concerning the utilization of paraffin wax shower for different sorts of conditions including post horrendous firm joints. 6 The wax mollifies the dry skin making it supple and warmth expands blood move through skin. Wax treatment likewise invigorates the perspiration organ, particularly those which hold their nerve supply.7 The paraffin wax shower is regularly utilized as powerful solution for improve flow and 


\section{PHARMACEUTICAL RESEARCH}

advances unwinding. Hands and feet are most regular sections to be treated with paraffin wax shower in exercise based recuperation.

\section{Execution}

Analyzed instances of post horrible firm lower leg exhibiting at Sajid Physiotherapy and Rehabilitation focus, Multan from March 2011 to February 2013, were surveyed by an orthopedic specialists. This investigation was a randomized controlled preliminary and thirty seven analyzed cases were enlisted and isolated in a control and study gathering. Gathering A had nineteen patients and Group B had 18 patients. The consideration criteria were age go from 20-60 years, torment, loss of ROM, with history of injury and break of lower leg. The patients with comparative protests however with careful treatment were barred. Gatekeepers and patients were educated about the advantages/dangers of the administration plans and educated assent was taken.

All the data was recorded and study factors were estimated at the standard including age, sexual orientation, foot included, torment force score, dorsiflexion, planterflexiom, reversal and eversion. The ROM was estimated by goniometer in recumbent position.9 Both gatherings were approached consistent schedule aside from Sundays, Group A patients were treated with joint assembly procedures and paraffin wax shower. While Group B patients were treated with just joint assembly methods. Wax treatment was given with paraffin wax shower machine by EnrafNonius, Netherlands. Paraffin wax is strong at room temperature and starts to soften over 37 $\mathrm{C}(99 \mathrm{~F})$ and liquefying point is 46 to $68 \mathrm{C}$ (115 to 154F). The paraffin wax shower was connected 25 minutes preceding joint preparation procedures including floats of the articular surface in prostrate position. The joint activation grade - 1 and grade 2 were usedfor torment the executives and unwinding, while Grade 3 were Used to stretch joint structures and increment joint play. 


\section{PHARMACEUTICAL RESEARCH}

\section{Results}

Age of the patient was 20 - 56 years in gathering An and in gathering B 21-62 years. Mean age of the patients was $36.43 \pm 0.8$ in gathering An and in gathering B was $41.33 \pm .07$ years. Thirty seven patients with post awful firm lower leg were enlisted and arbitrarily isolated in a control and study gathering. Gathering A had nineteen patients and Group B had 18 patients and treated for ten weeks. There were 12 male and 7 female patients in gathering An and 10 male and 8 female in gathering $B$. Toward the beginning of treatment the fundamental trademark were comparative in both the gatherings. Deficiencies in dorsiflexion, planterflexion, reversal, eversion agony and solidness were estimated when the treatment time frame. The control gathering was estimated at relating times. Relief from discomfort was discovered better in the two gatherings which were considered measurably critical with $p=0.001$, bunch $A(1.135 \pm 0.359)$ versus bunch $B(1.135 \pm$ 0.359). ROM in pre and post treatment degrees demonstrated that dorsiflexion was fundamentally expanded in gathering $A(1.135 \pm 0.359)$ versus bunch $B(1.135 \pm 0.0359)$.) and planterflexion was in gathering $A(1.337 \pm 0.422)$ versus bunch $B(0.841 \pm 0.264)$. Practical development indicated improvement in reversal in gathering $A(0.875 \pm 0.276)$ versus bunch $B$ $(0.966 \pm 0.305)$ and in eversion in gathering $A(0.948 \pm 0.300)$ versus bunch $B(0.674 \pm 0.213)$.

\section{Exchange}

Over numerous decades assembly procedures were utilized for increment of joint range yet now a days paraffin wax shower is additionally being utilized as elective treatment in post horrible hardened joints. 4 Various reports have been distributed, about activation strategies with paraffin wax shower as another treatment alternative in the administration of post awful firm joints 10 The general aftereffects of this investigation demonstrated the improved lower leg capacity and prompt relief from discomfort in gathering A. Studies presume that paraffin shower treatment isn't viable when utilized alone (for example at the point when not combined with remedial exercise). One such investigation was done, to make rules for thermotherapy intercessions for grown-up patients with RA, by Members OP et al. they found no factually noteworthy distinction for patients who had wax connected to the hand and lower leg versus a control after one month.10 It was, nonetheless, demonstrated that wax joined with exercise versus a control has demonstrated clinically significant advantages. 


\section{PHARMACEUTICAL RESEARCH}

\section{End}

Joint assembly and wax shower treatment is a powerful and gainful device to improve the side effects and personal satisfaction when contrasted with wax treatment alone. The joint activation and wax shower mediation effectively upgraded patient-arranged and clinician-situated proportions of capacity.

\section{References}

1. Neumann DA. Lower leg and foot. In Neumann DA: Kinesiology of the Musculoskeletal System: Foundations for Physical Rehabilitation. Philadelphia: Mosby. Chp. 14, $2009 .$.

2. Vora A, Myerson M. The average way to deal with triple arthrodesis. Sign and strategies for the executives of unbending valgus distortions in high hazard patients. Foot Ankle Clim. 2006.

3. Duray V. Physiotherapy and Rehabilitation, volume 1, second version; 2009 O'Brien T, Vicenzino B. An investigation of the impacts of Mulligan's assembly with development treatment of parallel lower leg agony utilizing a contextual investigation structure. Man Ther. 1999.

4. Vicenzino B. An investigation of the impacts of Mulligan's assembly with development treatment of horizontal lower leg agony utilizing a contextual analysis structure. Man Ther. 1999.

5. Portney L, Harris B. Impact of joint activation on joint solidness and dynamic movement of the metacarpal phalangeal joint. J Orthopedic Sports Med. 2011.

6. Marks R. Viability of paraffin wax showers for rheumatoid joint hands. Physiotherapy. 2000.

7. National Physical Laboratory. "Mechanical properties of materials". Kaye and Laby Tables of Physical and Chemical Constants; 2013. 